\title{
Processing of complex auditory stimuli (tunes) by rats and monkeys (Cebus apella)
}

\author{
M. R. D'AMATO and DAVID P. SALMON \\ Rutgers University, New Brunswick, New Jersey
}

\begin{abstract}
The degree to which rats and monkeys base their discriminations of complex auditory stimuli ("tunes") on frequency contours rather than on local features was investigated. In Experiment 1, groups of rats and monkeys trained with tunes as S+ and S- acquired a simple operant discrimination no faster than groups that received the same notes of each tune but in a new random order on each trial; neither did the groups differ on two transfer tests devised to detect learning of frequency contour in the tune-trained animals. Acquisition in the tune-trained and randomnotes groups seemed to be based on the overall frequency difference between $\mathbf{S}+$ and $\mathbf{S}-$, which was about 1.5 octaves. In Experiment 2, S+ and S- were similar to each other with regard to overall frequency and individual notes, the most salient differentiating characteristic of the tunes being their tonal pattern. The tune-trained groups were clearly superior to the randomnotes animals in acquisition, and an initial transfer test suggested that the former might have learned the discrimination on the basis of frequency contour. However, the detailed transfer tests of Experiment 3 strongly suggested that the tune-trained rats and monkeys based their discriminations primarily on local cues rather than on frequency contour. Based on the results of Experiment 4, the data of an earlier study that suggested frequency contour learning in monkeys and rats were reinterpreted in terms of control by local cues.
\end{abstract}

In a previous paper (D'Amato \& Salmon, 1982), we noted that monkeys seem to suffer a certain degree of modality asymmetry in their cognitive capacities, with vision holding a dominant position over audition. Thus, most of the laboratory-based information that we have about the monkey's learning, memorial, and conceptual abilities has been derived from vision-based studies. The lack of similar information in the auditory modalitiy is not for want of trying. The fact is that, compared with their performance on comparable visual tasks, monkeys have often proven relatively indisposed to learn either simple or complex relations based on acoustic cues (see Cowey, 1968; D'Amato, 1973; D'Amato \& Salmon, 1982; Dewson, 1979; Thompson, 1980, 1981; Wegener, 1964). Interestingly, the dolphin shows the reverse pattern, its cognitive capacities finding freer expression in the auditory than in the visual modality (Herman, 1980).

Given the highly developed auditory cortex of monkeys and the reports of their rich communication systems based on acoustic signals (see Marler, 1977; Snowdon, 1983), we thought their auditory cognitive capacities might have been underestimated by the general use of simple rather than temporally patterned acoustic stimuli for which they might have evolved

This research was supported by National Science Foundation Grant BNS-8207146. We wish to thank Paul Van Sant and Laura Buttles for their valuable help -with data collection. The authors' mailing address is: Department of Psychology, Busch Campus, Rutgers University, New Brunswick, New Jersey 08903. neural specializations. As pointed out by Herman, Richards, and Wolz (in press), the dolphin fares much better with "dynamic" than with "static" visual cues, which might be an analogous situation. In any case, we trained monkeys on a simple operant discrimination based on tunes-computer-generated patterned sequences of notes (D'Amato \& Salmon, 1982). All of the nine monkeys acquired the discrimination, and those that were tested showed substantial generalization across intensity and octave transformations. However, rats learned the same task much faster than the monkeys and showed similar generalization to the various transformations of the acoustic stimuli. Still, the pattern of results observed on the transfer tests and the fact that control rats trained with tones learned much more slowly than the tune-trained rats led us to conclude that the tunetrained rats and monkeys based their discriminations to some degree on the structures of the tunes.

One disconcerting note remained, however. If both species had detected and processed the temporal structure of the tune stimuli, why were the monkeys, with their more highly developed auditory cortex, so inferior to the rats in acquisition? More definitive evidence regarding the extent to which monkeys and rats are capable of detecting the pattern of notes of a tune was needed. To what degree does each species organize the notes of a tune into a tightly integrated unit based on the "frequency envelope," as humans do, and to what degree do they respond simply on the basis of local features? The present experiments were addressed to these issues. 


\section{EXPERIMENT 1}

Two 10-note tunes were constructed that had different mean frequencies and formed different patterns of notes. To humans, at least, the tunes were more easily differentiated on the basis of their patterns than by the difference in mean frequency. If rats and monkeys were sensitive to the temporal pattern of notes in a tune, then a group of animals trained with tunes should learn the discrimination faster than a group trained with the same notes but rearranged in a new random order on each trial. Moreover, after acquisition was achieved, the performance of the tune-trained animals should suffer when transferred to the random-notes condition, whereas the randomnotes group should generalize the discrimination to the tunes, which simply represented one possible randomization. Finally, if the tune-trained group had the mean frequency difference between $\mathrm{S}+$ and $\mathrm{S}-$ reversed while leaving the patterns intact, they should be far less disturbed by this "partial reversal" than the random-notes group, which would experience only the frequency reversal. On the other hand, to the extent that the tune-trained animals failed to make use of the patterns available to them, their behavior would be similar to that of the random-notes group.

\section{Method}

Subjects. The monkey subjects were laboratory-born, experimentally naive Cebus apella, three males and one female, between 24 and 49 months of age at the start of the study. They were obtained from another laboratory about 6 months before the beginning of the study. They were housed in group cages until the study began, at which time they were transferred to individual living cages. Throughout the experiment, they were maintained at $90 \%$ $100 \%$ of their full-ration body weights.

Twenty female Sprague-Dawley rats, obtained from Blue Spruce Farms, also served as subjects. They were between 145 and 265 days of age at the start of the study and were maintained at $80 \%$ $85 \%$ of their ad-lib weights (228-350 g). All had been control subjects in a previous, unpublished, T-maze study of conditioned taste aversion.

Apparatus. The monkey apparatus has been described in detail (D'Amato \& Salmon, 1982). In the present study, as in the earlier work, the right lever was employed, but the acoustic stimuli were presented by a speaker (10-W, 10-cm, 8-ohm Realistic Model FE103, distributed by Radio Shack) located directly below the lever rather than at the top of the chamber. This arrangement has been found to facilitate auditory discrimination learning (D'Amato \& Salmon, 1982; Segal \& Harrison, 1978). A display unit above the lever was illuminated at the onset of an S+ or S- trial and remained lit until the trial terminated.

Two of the rat apparatuses (standard operant testing chambers) were the same as those employed in the D'Amato and Salmon (1982) study, except that the speaker (also located below the response lever) was replaced with the same type as those used in the monkey chambers. A third chamber, similar to but larger than the first two, was fitted with the same speaker below the manipulandum.

The tunes delivered to each chamber were generated by a PET computer (see D'Amato \& Salmon, 1982) and amplified by the amplifier portion of an MTU D/A converter (Model K-1002). The high-frequency (HF) tune consisted of 10 monotonically decreasing frequencies, with note duration increasing monotonically from .07 to $.27 \mathrm{sec}$. The 10 frequencies were $4717,4032,3521$, $3125,2809,2632,2359,2101,1969$, and $1761 \mathrm{~Hz}(\operatorname{mean}=2902 \mathrm{~Hz})$. (Due to a programming error, the notes of the HF stimulus presented to the random-notes groups were slightly different, resulting in a mean frequency of $2743 \mathrm{~Hz}$.) The 10 notes of the lowfrequency (LF) tune were organized as five pairs of notes, with the frequency of the second member of a pair higher than the first and the frequency of each successive pair higher than the preceding pair. The frequencies were $496,822,539,947,590,1116,651$, 1359,727 , and $1736 \mathrm{~Hz}$ (mean $=898 \mathrm{~Hz}$ ). The duration of the first member of a pair was always $.07 \mathrm{sec}$, whereas the duration of the second was .27 sec. Each tune was therefore well structured with regard to frequency and note duration, and the patterns of the tunes differed sharply. Short, imperceptible off periods separated adjacent notes. One playing of a tune lasted about $2.0 \mathrm{sec}$, successive playings of a tune being separated by an interval that varied randomly between .5 and $1.5 \mathrm{sec}$. In the random-notes condition, on each trial the computer chose a new randomized order of the 10 notes, which remained fixed for that trial.

The intensities of the auditory stimuli were measured with a Simpson sound-level meter, Model 886, on the $C$ scale. In the monkey chambers, the meter was located directly in line with, and $12 \mathrm{~cm}$ from, the speaker; in the rat chambers, because of space limitations, it was oriented about $45 \mathrm{deg}$ with regard to the speaker and $5 \mathrm{~cm}$ away. The tunes measured maximum readings of 85 $87 \mathrm{~dB}$ (re $.0002 \mu \mathrm{bar}$ ) in the monkey chambers and $93-95 \mathrm{~dB}$ in the rat boxes.

During the reversal phase of the study, the notes of the HF tune were dropped 2 octaves and those of the LF tune were raised by 1.2 octaves. As a result, the mean frequency of the monotonically decreasing tune, which had been 1.6 octaves above the mean frequency of the second tune, was now 1.5 octaves below it (mean frequencies of 726 and $2178 \mathrm{~Hz}$, respectively).

Procedure. Five rats and one monkey were assigned randomly to the HF-tune, LF-tune, HF-random-notes, and LF-randomnotes conditions, where HF and LF indicate the stimulus that served as $\mathrm{S}+$. For the HF conditions, the corresponding LF stimulus served as $\mathbf{S}$-, and vice versa. (One tune-trained rat died after completing acquisition.) The same shaping procedure was used with the monkeys and rats. The animals were shaped to press the right lever, which was illuminated by a display unit in the case of the monkey chambers and a white-jeweled pilot light in the case of the rats. During two 48-trial sessions, the intertrial interval (ITI) and the duration of the fixed-interval (FI) reward schedule were gradually increased to 10 and $7 \mathrm{sec}$, respectively. On the third and fourth sessions, the ITI and FI schedule were increased to their terminal values, 30 and $10 \mathrm{sec}$, respectively (the same parameters as used in D'Amato and Salmon, 1982), and the S+ tune was played on all trials.

Discrimination training began with the fifth session, with the $S$ - tune introduced on 24 of the 48 trials, quasi-randomly intermixed. On an S+ trial, the lever light illuminated simultaneously with the playing of the positive tune (or randomized notes). Both stimuli continued until the animal completed the FI 10-sec schedule, which produced a food reinforcer (a 190-mg Noyes banana pellet for the monkeys and a $45-\mathrm{mg}$ pellet for the rats) and terminated the visual and acoustic cues. On $S-$ trials, if the animal failed to press the lever for $10 \mathrm{sec}$ after the trial's beginning, the trial terminated. Responding on $\mathrm{S}-$ trials was penalized by having each response extend the $S-$ duration for an additional $1 \mathrm{sec}$. Two monkeys served in the tunes condition and two in the randomnotes group; there were 10 rats in each condition. For half of each group the HF auditory stimulus (tune or random notes) served as $\mathrm{S}+$, and for half it was the LF. Acquisition continued until a discrimination ratio (DR) of .9 or greater was achieved in two successive sessions; in addition, to facilitate learning curve analysis, all subjects remained on acquisition for a minimum of 12 sessions. The DR was defined as the number of $S+$ responses executed during a session divided by the sum of $S+$ and $S-$ responses. Responses were recorded only during the first $10 \mathrm{sec}$ of an S+ or an S- trial. 
After completion of acquisition, a single transfer test session was given in which the tune animals were shifted to the randomnotes condition and the random-notes animals were shifted to the tunes.

All subjects were then returned to their original training condition (baseline) until a DR of .9 or better was attained for one session (which required no more than two sessions). Following this there were eight sessions of reversal training which employed the modified auditory stimuli described above. Because the random-notes group could not have based its discrimination on pattern, it faced a conventional reversal situation. For the tune animals, however, although the difference in mean frequency of the tunes was reversed, the patterns remained unaltered. If, for example, the monotonically decreasing tune had been $\mathrm{S}+$, it remained so, albeit with low- rather than high-frequency notes.

\section{Results and Discussion}

Turning to the rat subjects first, the results of the tune and random-notes groups over the first 12 acquisition sessions appear in Figure 1. An ANOVA based on condition (tune vs. random-notes), $\mathrm{S}+$ stimulus (HF vs. LF), and sessions (12) revealed that the consistent, but small, difference in favor of the tune-trained rats was not statistically significant $[F(1,16)=1.30]$. $S+$ stimulus was also insignificant $[F(1,16)<1]$, as were all of the interactions. The only significant result was sessions $[F(11,176)=68.00$, $\mathrm{p}<.0001]$. Analysis of the mean number of sessions to criterion, 10.4 and 14.0 (including the two criterial sessions) for the tune and random-note groups, respectively, also indicated comparable acquisition in the two groups of rats $[t(18)=1.33]$.

The mean DR on the transfer session, in which the tune rats were shifted to the random-notes condition and vice versa, was .90 for the tune rats and .96 for the random-notes group, a statistically insignificant difference $[t(17)=1.82]$. And, as suggested by $\mathrm{Ta}$ ble 1, there was no significant difference between the

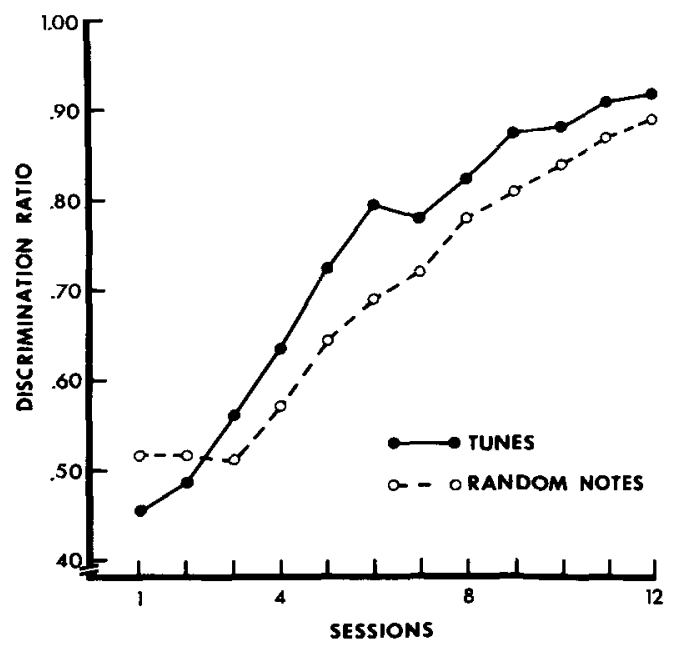

Figure 1. Mean discrimination ratios for the tune-trained and random-notes rats over the first 12 acquiaition sessions of Experiment 1.
Table 1

Mean Numbers of Sessions to Acquisition and Discrimination Ratios During Transfer and Reversal Tests for the Tune and Random-Notes Groups

\begin{tabular}{|c|c|c|c|c|c|c|c|c|}
\hline & \multirow{2}{*}{\multicolumn{2}{|c|}{$\begin{array}{l}\text { Mean Sessions } \\
\text { to Acquisition }\end{array}$}} & \multirow{2}{*}{\multicolumn{2}{|c|}{ Transfer }} & \multicolumn{4}{|c|}{ Reversal } \\
\hline & & & & & \multicolumn{2}{|c|}{ Session 1} & \multicolumn{2}{|c|}{ Session 8} \\
\hline & Tune & Rnd & Tune & Rnd & Tune & Rnd & Tune & Rnd \\
\hline $\begin{array}{l}\text { Rat } \\
\text { Mon }\end{array}$ & 170 & 0 & $\begin{array}{l}.90 \\
84\end{array}$ & .96 & $\begin{array}{r}.38 \\
35\end{array}$ & .30 & .57 & $\begin{array}{l}61 \\
67\end{array}$ \\
\hline Mon & 17.0 & 16.0 & .84 & .95 & .35 & .27 & .58 & .67 \\
\hline
\end{tabular}

tune and random-notes rats in reversal performance, either in Session 1 [t(17) $=1.61]$ or in Session 8 [t(17) $=.93$ ]. In short, there is no evidence from acquisition or the transfer and reversal tests that the tune-trained rats learned anything about the structure of the tunes.

The tune-trained and random-notes monkeys were closely comparable in acquisition, the two monkeys of the first group reaching criterion in 15 and 19 sessions and the latter two in 14 and 18 sessions. On the transfer test, the DRs of the two tune monkeys were .79 and .88 , and the values for the random-notes monkeys were .90 and 1.00 , not a very striking difference. With regard to reversal, the tune monkeys managed DRs of .34 and .36 on the first reversal session and .56 and .60 on the eighth. The corresponding values for the random-notes monkeys were .34 and .19 in Session 1 and .77 and .57 in Session 8.

In summary, except for speed of initial acquisition, which once again was faster in the rats $(p=.05$ by Fisher's exact test), the rats and monkeys produced virtually the same pattern of results (see Table 1). Neither species appears to have paid much heed to the patterns of notes available in the tunes. Their discriminations were apparently based on the overall frequency difference between the tunes, a cue that was present to an equal degree in the random-notes condition.

To provide some indication of the animals' response rates, the mean numbers of responses executed during the first $10 \mathrm{sec}$ of $\mathrm{S}+$ and $\mathrm{S}-$ trials were obtained for the two critical sessions of acquisition. As in the D'Amato and Salmon (1982) study, there was considerable intersubject variability but no evidence that speed of acquisition was related to response rate, and the mean response rates of the rats $(\mathrm{S}+$ rate $=4.24, S-$ rate $=.30$ ) were higher than those of the monkeys (3.10 and .21, respectively).

One possible reason why the tune-trained animals failed to detect the structure of the tunes is that the large difference in the tunes' mean frequency simply overshadowed pattern cues. While the latter are the dominant cues for human observers, animals may rely more heavily on nonconfigural properties. The next experiment tested this hypothesis by employing tunes that were closely similar except for the pattern of their notes. 


\section{EXPERIMENT 2}

Two 7-note tunes were generated that were very similar with respect to their mean frequency and component notes but noticeably different in their patterns. Both began with the same note, and 4 of the 7 notes appeared in both tunes. Tonal pattern thus seemed to be the dominant differentiating property of the tunes. The tune-trained and random-notes animals that acquired the discrimination were given a transfer test in which the tune animals were shifted to the random-notes condition and vice versa.

\section{Method}

Subjects. The monkey subjects were four laboratory-born, experimentally naive Cebus apella, two males and two females, between 28 and $\mathbf{5 6}$ months of age at the start of the study. They were obtained at the same time and from the same source as the monkeys of Experiment 1. During the study, they were maintained at $88 \%-100 \%$ of their full-ration body weights.

Eight experimentally naive female rats of the type used in Experiment 1 served. They were about 285 days of age at the start of the study, and were maintained at $80 \%-85 \%$ of their ad-lib weights (284-325 g).

Apparatus. The apparatuses of Experiment 1 were employed in the present study, except that the third, larger, rat chamber was not used.

The notes of tune 1 formed a distinctive pattern of alternating increases and decreases in frequency: 1397, 1572, 1397, 1572, 1984, 2083 , and $1046 \mathrm{~Hz}$ (mean $=1579 \mathrm{~Hz}$ ). The pattern of tune 2 was a decrease in frequency followed by a monotonic increase: $1397,1173,1316,1397,1572,1761$, and 1984 (mean = $1514 \mathrm{~Hz}$ ). Note duration varied between .07 and $.25 \mathrm{sec}$, with the duration of the tune being about $1.3 \mathrm{sec}$. The randomization program employed caused the duration to be somewhat longer in the randomnotes condition, about $1.6 \mathrm{sec}$. The intensities of the tunes were in the same range as those reported in Experiment 1.

Procedure. Two rats and one monkey were assigned randomly to four conditions: tune 1, tune 2, random notes 1, and random notes 2 , where 1 and 2 indicate the auditory stimulus that served as S+. Tune 2 was $S-$ for condition tune 1 , tune 1 for tune 2 , and so on. The shaping and training procedures were the same as in Experiment 1, except for the following. On Acquisition Day 9, in an attempt to increase attention to the auditory stimuli, the light above the response lever no longer was illuminated during a trial. To discourage responding on S- trials, on Day 36 the penalty was changed so that the $S-$ stimulus continued to play for $10 \mathrm{sec}$ after the last $\mathbf{S}-$ response. These appeared to be useful modifications, although we have no way of objectively evaluating their impact.

As in the previous experiment, the acquisition criterion was two successive sessions with DR of .9 or better. The tune-trained animals that met criterion were given a single test session with random notes, and the one successful animal in the random-notes condition was tested with the tune stimuli.

\section{Results and Discussion}

None of the four rats in the random-notes condition showed the slightest sign of learning the discrimination. Figure 2 reveals that at the end of 75 sessions (3,600 trials) they were still responding at a chance level, at which point they were terminated. Figure 2 also presents the results of one random-notes monkey (Tiny) that learned the discrimination (in 88 sessions). The other monkey (Slim) was terminated after 80 sessions because of his flat, though above-chance performance. A t test based on the DRs of Sessions 66-

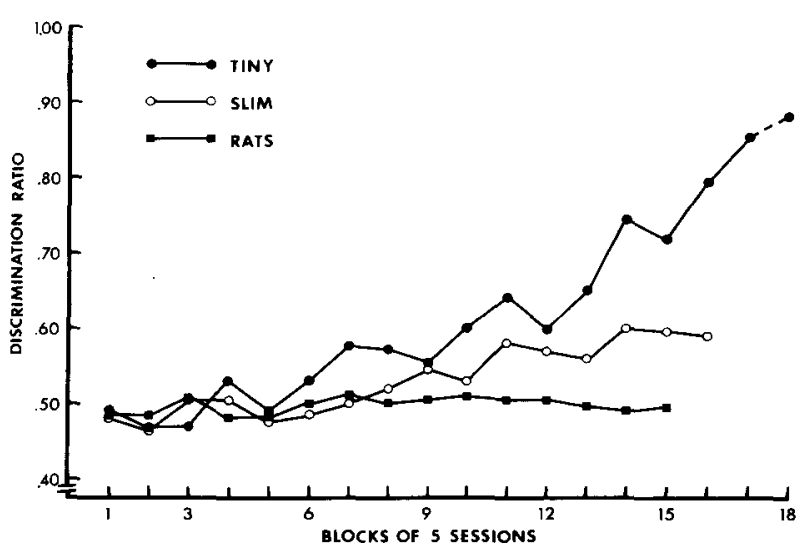

Figure 2. Mean discrimination ratios for the four random-notes rats and two random-notes monkeys of Experiment 2 in blocks of five seasions. The last date point of Tiny's curve is based on three sesalons and includes the two criterlal seasions.

75 showed Slim's mean DR to be significantly greater than $.50[t(9)=3.69, p<.01]$, whereas none of the mean DRs of the four random-notes rats were significantly above chance. It seems clear from these data that the monkeys fared much better than the rats when faced with the random-notes discrimination.

Both tune-trained monkeys learned the discrimination, Goldy in 43 sessions and Flo in 62 . On the other hand, only two of the four tune-trained rats clearly reached acquisition criterion, and they required 98 and 125 sessions. One tune rat was terminated after 100 sessions, at which point it was responding at a chance level. The response rate of the fourth rat in the tune group declined to a very low level, with responses occurring on only a small number of trials. Although it nominally met the acquisition criterion (in only 49 sessions), it executed a total of only 15 responses during the first criterial session and a mere 6 responses during the second. Moreover, during 35 postcriterial sessions, it never again met criterion, its DRs fluctuating wildly between 0 and 1.0 because of its extremely low response rate. If we dismiss this rat's results, the superiority of the monkeys over the rats in the tune discrimination is quite clear.

Transfer test. The results of the transfer test, in which the tune animals were shifted to the randomnotes condition and the random-notes monkey was shifted to the tunes, were substantially different from those of Experiment 1. Tiny, the random-notes monkey, completely generalized the discrimination to the tune stimuli (DR = .92), but Goldy and Flo showed little or no generalization when shifted to random notes (DRs of .72 and .57, respectively). However, the tune rats generalized even less to the randomnotes conditions (DRs of .67 and .44).

Although acquisition was considerably more prolonged in this experiment than in the previous one, the rats' mean response rates on the two criterial ses- 
sions were comparable to those of Experiment 1 $(S+$ rate $=4.63, S-$ rate $=.40)$. On the other hand, by the end of acquisition the response rates of the three monkeys that reached criterion had declined well below those observed in Experiment 1 (S+ rate = $1.65, \mathrm{~S}-$ rate $=.14$ ).

The acquisition results of the present experiment represent the first time in our experience that monkeys were superior to rats in acquiring an auditory discrimination. Both tune monkeys learned the discrimination and in only about half the number of sessions required by the (two of four) tune-trained rats that clearly acquired the task. Even more impressive is the difference between the random-notes groups. None of the four rats in this condition was responding above chance at the end of 75 sessions. However, the performance of both random-notes monkeys was significantly above chance, and one monkey went on to reach criterion in fewer sessions than either of the successful tune rats. It appears from the present experiments and our earlier work that rats are more efficient than monkeys in detecting gross frequency differences between two auditory signals. When a finer analysis of the acoustic stimulus is demanded, however, the advantage shifts to the monkey.

But what is the nature of this finer analysis? It is not necessarily based on the patterns of the tunes, as the random-notes monkey that reached criterion did not have the benefit of such cues. Still, the possibility exists that the tune-trained monkeys, and perhaps the tune rats as well, based their discriminations on the temporal pattern of notes, which could account for their superiority over the random-notes subjects. Clearly, more information is needed regarding the specific cues upon which each animal formed its discrimination. The next experiment addressed this issue.

\section{EXPERIMENT 3}

The three monkeys and two rats of Experiment 2 that acquired the discrimination were tested on a variety of transformations of the acoustic stimuli in an effort to pin down the essential controlling cues. The experiment was quite prolonged, lasting between 7 and 8 months, and involved almost $\mathbf{4 0}$ separate tests. Because we had no clear idea of what we might find, the strategy was necessarily opportunistic, in that tests were devised to illuminate results obtained on previous assessments. To make the description of the experiment more manageable, tests that were redundant or uninformative will not be discussed.

\section{Method}

Procedure. Only certain general features of the procedure will be discussed here. To avoid unnecessary duplication, the specific transfer tests will be described in the Results and Discussion section. In most cases, after completion of a transfer test, the animals were returned to the baseline discrimination until a specified criterion was met before being advanced to the next transfer test. This necessary procedure contributed considerably to the length of the experiment. Rat 46 , which required 125 sessions to meet the acquisition criterion, had difficulty recovering baseline and was therefore given only a few transfer tests.

The training procedures and parameters were the same as those employed in Experiment 2 with the single exception that, because of the decreased response rates noted earlier, the monkeys' FI 10 -sec food schedule was changed to a variable-interval (VI) 7.5sec schedule. During each session, the same distribution of VI intervals (between 6 and $10 \mathrm{sec}$ ) occurred on $S+$ and $S-$ trials. This schedule change was introduced after the first few transfer tests and resulted in a moderate increase in response rate. Because they maintained their response rates at a relatively high level, the rats' food schedule was not changed.

Five types of transformations of the acoustic stimuli were assessed. In the octave transformations, the acoustic stimuli were shifted down or up by 1 octave. The duration transformations either compressed or extended the duration of $S+$ and $S-$. In the third type of transformation, single notes were dropped from both $S+$ and $S-$ (e.g., the first note of each). Changing notes of $S-$ to make them identical to the corresponding notes of $S+$ was the fourth type of transformation. Finally, multiple notes were dropped from $S+$ and $S-$, in the extreme case dropping all but one. In general, the temporal order in which the various transformations occurred corresponds to the above sequence.

\section{Results and Discussion}

Baseline recovery. The criterion for recovery of baseline varied from three successive sessions with DRs of .85 or greater to a single session with a DR of at least .9 , but the same criterion was always applied to the monkeys and rats. The mean numbers of sessions required to recover baseline (including the criterial sessions) were $2.66,1.76$, and 2.28 for Goldy, Flo, and Tiny, in that order. Rat 41 averaged 5.10 sessions, and, over its three baseline recoveries, Rat 46's average was 16.0 . Thus, baseline recovery was substantially faster in the monkeys than in the rats.

Octave transformations. Table 2 presents the results of the transfer tests, in the order in which they were administered. During all transfer tests, the customary reinforcement schedules remained in effect with the transformed stimuli. In the first transfer test, the notes of S+ and S- were lowered by 1 octave, that is, the frequency of each note was halved. Three test sessions were given; to conserve space, the results of Sessions 1 and 3 only are shown (in rows 1 and 2 of the table). The two tune-trained monkeys, Goldy and Flo, showed little transfer, even in the third session, when their DRs were .54 and .53 , respectively. The two tune-trained rats fared no better. managing DRs of only .52 and .39 in the third session. This was the first indication that the tune-trained animals might have learned little about the patterns of the tunes. If they had, considerable generalization on the octave transformation would be expected, at least by the second or third session (see Blackwell \& Schlosberg, 1943). Tiny was not expected to show much transfer because he was trained and tested with random notes and therefore did not have any pattern cues with which to mediate generalization. 
Table 2

Discrimination Ratios on the Transfer Tests of Experiment 3

\begin{tabular}{|c|c|c|c|c|c|c|}
\hline Test & Row & \multicolumn{5}{|c|}{ Subject } \\
\hline $\begin{array}{l}\text { Lowered } 1 \text { Octave } \\
\qquad \mathrm{S}^{-}, \mathrm{S}-\end{array}$ & $\begin{array}{l}1 \\
2\end{array}$ & $\begin{array}{l}.37 \\
.61\end{array}$ & $\begin{array}{l}.65 \\
.54\end{array}$ & $\begin{array}{l}.53 \\
.53\end{array}$ & $\begin{array}{l}.50 \\
.52\end{array}$ & $\begin{array}{l}.50 \\
.39\end{array}$ \\
\hline S+ Only & $\begin{array}{l}3 \\
4\end{array}$ & $\begin{array}{l}.92 \\
.78\end{array}$ & $\begin{array}{l}.50 \\
.64\end{array}$ & $\begin{array}{l}.80 \\
.85\end{array}$ & $\begin{array}{l}.90 \\
.92\end{array}$ & $\begin{array}{l}.53 \\
.56\end{array}$ \\
\hline $\begin{array}{c}\text { Duration (sec) } \\
.75 \\
2.10\end{array}$ & $\begin{array}{l}7 \\
8\end{array}$ & -- & $\begin{array}{l}.95 \\
.94\end{array}$ & $\begin{array}{l}.86 \\
.87\end{array}$ & $\begin{array}{l}.92 \\
.83\end{array}$ & - \\
\hline Note 7 Deleted & $\begin{array}{r}9 \\
10\end{array}$ & $\begin{array}{l}.93 \\
.94\end{array}$ & $\begin{array}{l}.49 \\
.60\end{array}$ & $\begin{array}{l}.80 \\
.84\end{array}$ & $\begin{array}{l}.58 \\
.46\end{array}$ & $\begin{array}{l}-- \\
--\end{array}$ \\
\hline S+, S- Raised 1 Octave & $\begin{array}{l}15 \\
16\end{array}$ & $\begin{array}{l}.42 \\
.56\end{array}$ & $\begin{array}{l}.48 \\
.57\end{array}$ & $\begin{array}{l}.75 \\
.96\end{array}$ & $\begin{array}{l}.52 \\
.50\end{array}$ & -- \\
\hline $\begin{array}{l}\text { Notes of S-Made Same Fre } \\
\quad 7\end{array}$ & $\begin{array}{l}17 \\
18\end{array}$ & $\begin{array}{r}.88 \\
1.00\end{array}$ & $\begin{array}{l}.46 \\
.71\end{array}$ & $\begin{array}{l}.63 \\
.67\end{array}$ & $\begin{array}{l}.53 \\
.58\end{array}$ & $\begin{array}{l}-- \\
--\end{array}$ \\
\hline 6 & $\begin{array}{l}19 \\
20\end{array}$ & $\begin{array}{l}.76 \\
.67\end{array}$ & $\begin{array}{l}.88 \\
.87\end{array}$ & $\begin{array}{l}.94 \\
.94\end{array}$ & $\begin{array}{l}.80 \\
.93\end{array}$ & $\begin{array}{l}-- \\
--\end{array}$ \\
\hline 6 and 7 & $\begin{array}{l}21 \\
22\end{array}$ & $\begin{array}{l}.64 \\
.71\end{array}$ & $\begin{array}{l}.50 \\
.62\end{array}$ & $\begin{array}{l}.46 \\
.48\end{array}$ & $\begin{array}{l}.59 \\
.59\end{array}$ & $\begin{array}{l}-- \\
--\end{array}$ \\
\hline $1-5$ & $\begin{array}{l}23 \\
24\end{array}$ & $\begin{array}{l}.83 \\
.83\end{array}$ & $\begin{array}{l}.81 \\
.72\end{array}$ & $\begin{array}{r}1.00 \\
.93\end{array}$ & $\begin{array}{l}.84 \\
.77\end{array}$ & $\begin{array}{l}-- \\
--\end{array}$ \\
\hline Note 7 Only & 32 & .59 & 1.00 & .90 & 1.00 & -- \\
\hline
\end{tabular}

${ }^{*} S+=$ Tune 1 .

In the next octave transformation, $\mathrm{S}+$ was lowered 1 octave while $S$ - remained undisturbed; this was followed by the reverse manipulation, $S-$ being selectively lowered 1 octave. Three test sessions were given on each transformation; the first and third sessions appear in Table 2. Rows 3-6 show that some animals suffered more from the first transformation than from the second. Goldy and Rat 46 did very poorly when the frequency of $S+$ was lowered, showing little recovery over the three sessions, but when S- only was lowered 1 octave, Goldy transferred completely in the first session and the rat reached a high level of responding in the last two test sessions. The other two tune-trained animals, Flo and Rat 41, performed poorly in the first session with S- lowered, but recovered nicely over the next two sessions. Only
Tiny seemed not to exhibit a strong asymmetrical pattern of performance on the two transformations. These data suggest that most of the animals' discriminative behavior was under greater control by one stimulus than the other, $\mathrm{S}+$ in the case of Goldy and Rat 46, and S- in the case of Flo and Rat 41, which is another indication that the tune-trained animals did not learn a great deal about the patterns of the tunes.

Rows 12-14 show the results of applying the same octave transformations, one session on each, some 40-70 sessions later (by which time Rat $\mathbf{4 6}$ was terminated). Although the animals had by this time experienced a large number of transformations, their performance was generally similar to that obtained on the earlier tests. All animals performed very poorly 
when both stimuli were dropped 1 octave; Goldy's discrimination was still under greater control by $\mathrm{S}+$ than by $\mathrm{S}-$; and the opposite was true for Flo and Rat 41. At this point, Goldy and Flo were given a number of additional sessions with both tunes lowered an octave, to see whether their depressed performance would rebound quickly. At the end of seven such sessions, Goldy was still responding at a chance level, and it required 16 sessions for Flo's DR to reach .8.

All animals were then tested for two sessions with $\mathrm{S}+$ and $\mathrm{S}-$ raised 1 octave (rows 15-16). Only Flo performed at a high level, although her DR on the first 24 trials of the first session was .69. To what degree her performance represents rapid learning, perhaps due to the additional training with the lowoctave tunes, cannot be ascertained. But, in any case, the stark inability of the animals to generalize to a 1-octave transformation is indicated by the final test given near the end of the experiment. As shown in row 29 , with $S+$ and $S-$ again lowered 1 octave, only Flo, who received substantial training in this condition, responded above chance, and only moderately so at that.

We can conclude from the octave generalization tests that the tune-trained animals did not learn a sufficient amount about the structure of the tunes to permit substantial generalization when both tunes were simultaneously increased or decreased by 1 octave. Interestingly, starlings trained to discriminate rising from falling tonal patterns also failed to generalize to 1-octave transformations (Hulse, Cynx, \& Humpal, 1984).

Duration transformations. Rows 7-8 show that when the tunes, whose normal durations were about $1.3 \mathrm{sec}$, were either compressed to about $.75 \mathrm{sec}$ or expanded to $2.1 \mathrm{sec}$, the performance of the tune animals was virtually unaffected. (Tiny did not receive these tests). Because note duration was so ineffectual, at this point the duration of the notes was made equal at a value that maintained the total duration of $S+$ and $S-$ at about $1.3 \mathrm{sec}$. All animals generalized immediately to this transformation, with DRs ranging from .87 to 1.00 .

In a later series of tests, the duration of the auditory stimuli was reduced even further. With durations as short as $.4 \mathrm{sec}$, discriminative performance was unaffected in all animals. Duration had to be reduced to about $.3 \mathrm{sec}$ before a decrement was observed in some animals (row 11). Clearly, then, differences in note duration or in tempo played no role in the tune or the random-notes animals' discriminative behavior.

Single note deletion. In this series of single-session transfer tests, one note was deleted from both $\mathrm{S}+$ and $\mathrm{S}-$, always from the same ordinal position. Note 7 was dropped from both stimuli first, and row 9 indicates that the discriminative performance of two of the three tune animals suffered badly; Flo's loss was small. Tiny, the random-notes monkey, was completely unaffected by this transformation. Note 1 was next dropped from both stimuli; deletion of note 2 followed, and then note 3 . None of these alterations had any effect on performaance, the lowest DR observed being .85 . Note 7 was again deleted, with results similar to those obtained earlier (row 10). Later in testing, when we began to suspect that note 6 might be a dominant controlling cue for Tiny, we deleted it from both stimuli. The DRs of row 28 show that this manipulation exerted a negative effect on Tiny only.

The results of single-note deletion suggest, then, that note 7 of $S+$ and $S-$ was important controlling cues for at least two of the three tune animals, but not for Tiny, for whom the dominant note was 6 .

Changing $S-$ notes to $S+$. In the next series of transformations, by appropriate frequency changes, one or more notes of $S-$ were made identical to the corresponding notes of $\mathrm{S}+$, a manipulation that essentially degraded the information available in $\mathbf{S}-$. Because the first note of $\mathrm{S}+$ and $\mathrm{S}-$ was already identical, notes 2-7 of $S-$ were changed, one at a time, to be the same as the corresponding notes of $S+$; two test sessions were given with each manipulation. Performance was little affected when this manipulation was applied to notes 2-5; the lowest DR observed was .75. When note 7 was so transformed, however, an unmistakable decrement occurred in the three tune animals (rows 17-18), but not in Tiny. The reverse pattern of results was obtained when note 6 of $S-$ was changed in this manner (rows 19-20).

Notes 6 and 7 of $S+$ and $S-$ were then made identical, notes 1-5 retaining their original frequencies. Performance was depressed in all subjects, severely so in the three tune animals (rows 21-22). The complementary transformation, leaving intact notes 6 and 7 but making notes $1-5$ the same, had little, if any, effect, at least during the first test session (rows 23-24). But when notes 1-6 were made identical, Tiny's performance fell to near-chance levels, in sharp contrast to the tune monkeys, whose discriminations remained fully intact (rows 25-26). Rat 41, who was having trouble recapturing baseline during these tests, did not seem to be differentially affected by the last two transformations.

We may conclude from this series of tests that notes 1-5 played very little role in the discriminative behavior of any animal. Note 7 again seemed to carry a great deal of the discriminative burden for the tunetrained animals; note 6 seemed to do so for the randomnotes monkey.

Multiple note deletion. The aim of this series of manipulations was to see whether discriminative behavior could be sustained with only one or two notes in each stimulus. If, as conjectured, notes 6 and 7 were the important controlling stimuli, substantial 
generalization should occur to discriminative stimuli consisting solely of these notes. This proved to be the case in two separate tests that employed notes 6 and 7 only (rows 27 and 30).

Inasmuch as Tiny's discriminative behavior seemed to be largely under the control of note 6 , exposing all animals to notes 1 and 7 should have a depressing effect only on his performance. Row 31 indicates such a trend, though not strikingly so. The DRs of the first 24 of the 48 trials are perhaps more revealing. Tiny's DR was only .33 during these trials, whereas those of the three tune animals ranged between .98 and 1.00 .

The concluding transformations went all the way, reducing $S+$ and $S-$ to a single note. Tested with note 7 alone, the performance of the three tune animals under this extreme transformation was very high; in contrast, Tiny's performance was quite low (row 32). When tested with note 6 only, however, Tiny's DR rebounded to .96 (only Tiny received this test). These last tests are particularly interesting in that when the dominant controlling note of each stimulus was presented alone, discriminative behavior seemed to be potentiated, even in the rat. This suggests that the other six notes, far from forming part of a tonal pattern for the animals, detracted from the discriminability of the auditory stimuli.

Response rates. The similar results obtained with the tune-trained rats and monkeys emerged despite different food-reinforcement schedules and a sizable difference in response rates. For example, during the last three transfer tests (rows 30-32), the mean $S+$ and $S$ - rates were 5.53 and .30 , respectively, for Rat 41, whereas the corresponding mean values for Goldy and Flo were .89 and .05 . The latter $S+$ value is lower than usual for substantial DRs, but mean $S+$ rate rarely exceeded 2.0 for any of the three monkeys. Low DR values were often, but not always, the result of a large increase in $S-$ rate. For example, the $S+$ and $S-$ rates associated with the final 1-octave transformation (row 29) were 6.12 and 7.04 , respectively, for Rat 41 ; the corresponding mean $S+$ and $S$ - rates were .88 and .63 for the three monkeys.

The results obtained on the various transfer tests indicate that, in spite of more than 25,000 exposures to each tune, the tune-trained animals learned very little about the sequential pattern of notes. They never organized the notes of a tune into an integral unit based on the frequency envelope, the way humans do. Rather, they based their discriminations on local features of the tune stimuli. Two questions are immediately raised by this conclusion. Why, in Experiment 2 , did the tune-trained monkeys and rats acquire the discrimination so much faster than the corresponding random-notes animals, and why did they fail to transfer to the random-notes condition? The nature and location of note 7 of the tunes provide a reasonable answer to both of these questions. As it happened, the frequency difference between note 7 of S+ and note 7 of S- was the largest of any of the notes. In addition, being the final note of a tune might have set note 7 off from the others. Thus, a highfrequency note followed by offset of the tune could have served as one discriminative cue and a lowfrequency cue in the same location, as another. The location cue was not available for the subjects in the random-notes condition, which put them at a disadvantage during acquisition. On the other hand, reliance on this cue would cause a deterioration in performance when the tune animals were shifted to the random-notes condition.

The transfer data of Tiny, the random-notes monkey, provide additional support for this analysis. Because the notes of S+ and S- were separately randomized on each trial, one would not necessarily have expected note 7 (as defined by the tunes) to provide the major basis for discrimination, which was the case. Note 6 seemed to play this role, possibly because note 6 of the tune 1 was the highest frequency note of both stimuli.

By emphasizing the dominant controlling role of note 7 in the case of the tune-trained animals and note 6 for Tiny, we do not mean to imply that the animals' discriminative behavior was in all instances based solely on a single note. Flo's relatively high performance on the two tests in which note 7 was deleted (rows 9-10) suggests some control by other notes, possibly note 6 , at least in this point of testing. The important point is that in all animals a single note did exert overwhelming control, and where multiple sources of control might have operated, these very likely were also based on local features of the tunes.

It was pointed out earlier that certain findings of the D'Amato and Salmon (1982) study had indicated that their subjects' discriminations were probably based on the structure of the tunes. Given the present data, we are inclined to reinterpret those findings as follows. That the rats and monkeys might have learned the structure of the tunes was suggested primarily by (1) generalization of the discrimination across octave transformations, (2) substantial generalization to the first half but not to the second half of the tunes, and (3) the greatly inferior acquisition rate of rats trained with broken tones rather than with tunes. All of these outcomes are accountable in terms of the more local discriminative mechanisms employed by the subjects of the present study.

One of the tunes of the earlier research ("gliss") was approximately 2 octaves higher in median frequency than the other tune ("charge"). In the first octave transformation, the charge tune was raised 1 octave, causing virtually no disturbance in the discriminative behavior of the monkeys or the rats (mean DRs $=.89$ and .93 , respectively). Raising charge another octave did disrupt performance, but 
Table 3

Mean Numbers of Sessions to Complete Phase 1 and Reach Acquisition Criterion in Phase 2

\begin{tabular}{lccc}
\hline Experiment & Phase 1 & Phase 2 & Stimuli \\
\hline 4, Present study & 4.0 & 3.75 & Tones, 5 octaves apart \\
2, D'Amato \& Salmon, 1982 & 3.9 & 4.50 & Tunes, charge and gliss \\
3, D'Amato \& Salmon, 1982 & 3.2 & 15.00 & Tones, 2 octaves apart \\
\hline
\end{tabular}

a significant amount of generalization nevertheless occurred (mean DRs $=.69$ and .87 , respectively). Given what we know from the present experiments, it is reasonable to conjecture that the rats and monkeys of the earlier study based their discriminations primarily on the first note or two of the tunes, which formed the greatest frequency contrast (more than 4 octaves) and enjoyed a salient location cue. If such were the case, one would expect a high degree of generalization to the 1-octave transfer test because the initial notes were still separated by a substantial frequency difference (more than 2 octaves). A second doubling of the frequencies of the charge tune would narrow the difference considerably and hence lead to less generalization; the fact that the rats were less disturbed than the monkeys by this transformation can be attributed to their generally superior ability to make frequency difference determinations of this kind. This interpretation directly accounts for finding 2.

As for finding 3, two factors could have been responsible. First, considerably more habituation to the acoustic stimuli would occur in the tone control group. Of much more importance, the $S+$ and $S-$ tones were separated by approximately the same frequency difference that separated the median frequency of the tunes -2 octaves. But, as already indicated, the initial notes of the tunes were considerably further apart in frequency. Thus, a tune animal that based its discrimination on the first one or two notes would have a more salient frequency difference cue than that available to the tone rats. If this interpretation is correct, rats trained with tones based on only the first note of the charge and gliss tunes should learn the discrimination at least as fast as the tunetrained rats. Experiment 4 investigated this possibility.

\section{EXPERIMENT 4}

Every effort was made to duplicate the procedures employed with the broken-tone group in Experiment 3 of the D'Amato and Salmon (1982) study. In that study, the frequency of the HF tone was $1454 \mathrm{~Hz}$ and that of the LF tone was $363 \mathrm{~Hz}$, a difference of 2 octaves. In the present experiment, the frequencies of the HF and LF tones were 8929 and $243 \mathrm{~Hz}$, respectively, a difference of a little more than 5 octaves. The latter frequencies correspond to the first notes of the gliss and charge tunes used in Experiment 2 of D'Amato and Salmon (1982).

\section{Method}

Subjects and Apparatus. Four experimentally naive female rats, of the same type, and obtained from the same source, as those of Experiment 1, served, half with the HF tone as $\mathrm{S}+$ and half with the LF tone in this role. The rats were about 80 days of age at the beginning of the study and were maintained at $80 \%-85 \%$ of their ad-lib weights $(180-240 \mathrm{~g})$. The apparatus differed from that used in Experiments 2 and 3 of D'Amato and Salmon (1982) only with regard to the speakers, which were the same as those of Experiments 1-3 above. The same program was used to generate the tones as in the D'Amato and Salmon (1982) study; on an S+ or S- trial, the tone played for $1.87 \mathrm{sec}$, with successive playing separated by off times that varied randomly between .5 and $1.5 \mathrm{sec}$. The only difference was in the frequencies of the tones, which in the present study were 8929 and $243 \mathrm{~Hz}$.

Procedure. After shaping, which was virtually identical to that employed in the earlier study, the rats were exposed to Phase 1 , followed by Phase 2. In Phase 1, tones were presented on S+ trials only, $\mathrm{S}-$ trials being signaled solely by the lever light. Phase 1 training continued until a DR of $\mathbf{. 8 5}$ or better was achieved or for a maximum of five sessions. The S- tone was introduced in Phase 2 , which continued until the criterion of two consecutive sessions with DR of . 9 or better was met. These were the same procedures as those employed in the D'Amato and Salmon (1982) study.

\section{Results and Discussion}

Table 3 presents the results of the present study, and to facilitate comparison, the corresponding data from Experiments 2 and 3 of D'Amato and Salmon (1982) also appear in the table. The four rats of the present study required only $3,4,4$, and 5 sessions to reach criterion in Phase 2. (As in the D'Amato and Salmon, 1982, experiments, these values include only one of the two criterial sessions.) The mean, 3.75, compares favorably with the corresponding mean of D'Amato and Salmon's (1982) tune-trained subjects (4.50), and is a great deal less than the mean of their broken-tone group (15.0). As might be expected from the fact that the $\mathrm{S}-$ acoustic stimulus did not occur during Phase 1, performance was comparable in all groups during this phase.

From the present data, it appears that the transfer and related results of the tune-trained animals of D'Amato and Salmon (1982) can be accounted for by a discrimination based on local features of the tunes, that is, the frequencies of their initial notes.

\section{GENERAL DISCUSSION}

When presented with simple or complex acoustic stimuli that differ significantly in frequency, discriminative behavior develops rapidly in rats, considerably faster than in monkeys trained under comparable experimental conditions (Experiment 1; D'Amato 
and Salmon, 1982, Experiments 1 and 2). Being nocturnal animals, rats rely less on visual than on olfactory and auditory cues, and they make extensive use of ultrasound as infants, in mating, and in aggressive behavior (Sales \& Pye, 1974). Possibly their sensitivity to such a wide portion of the frequency spectrum predisposes them to attend to this dimension and make efficient use of information encoded as differences in frequency.

It is entirely another matter when the discriminative stimuli cannot be differentiated on the basis of gross frequency differences, as was the case in Experiment 2. Despite the benefit of 75 training sessions, none of the four random-notes rats showed the slightest sign of discriminating the acoustic stimuli. On the other hand, one random-notes monkey reached acquisition criterion and the other was responding well above chance at the 75-session mark. Whatever the acoustic analyzers might be that permitted the monkeys to discriminate between the two randomnotes stimuli, they obviously are either absent or less developed in rats. The tune-trained monkeys also proved superior to the tune-trained rats, not only in acquisition (Experiment 2), but also in recapturing baseline after transfer testing (Experiment 3).

The tune-trained monkeys and rats of Experiment 2 were alike in their apparent failure to extract frequency pattern, or contour, as an identifying feature of each tune. As already mentioned, to humans this is the dominant property of the acoustic stimuli, readily recognizable after octave generalizations but much degraded by transformations that left discriminative performance largely untouched in the animals. It should be noted that focusing on local features was not a particularly efficient way of solving the discriminative problem, either for the monkeys or the rats. The mean number of sessions to criterion for the two tune-trained monkeys was 52.5 , compared with only 17.0 for the two tune monkeys of Experiment 1 (who had the benefit of a mean-frequency difference cue); for the tune-trained rats, the corresponding values were 111.5 and 10.4 . That the rats failed to make use of frequency pattern is perhaps not surprising. The monkeys' failure is less easy to accept, given certain related data in the literature.

As noted by Miller (1977), many animals, raccoons to elephants, seem capable of learning responses to spoken commands. Appropriate responding despite intensity and frequency transformations imposed by the same or different speakers was reported more than 70 years ago (Shepherd, 1911, 1912), which suggests that the animals were responding to acoustic patterns in the speech sounds (common names, usually) rather than to limited local features. However, without a more detailed analysis of the controlling features of the acoustic signals, the latter possibility cannot be ruled out. More recently, Dewson, Pribram, and Lynch (1969) trained rhesus monkeys to discriminate between the vowel sounds [i] and [u], each having a duration of approximately $300 \mathrm{msec}$. The fact that the monkeys generalized the discrimination from a male to a female voice was regarded as evidence that the pattern of the speech sound was learned rather than any "particular constituent." While this may be the case, it should be noted that the fundamental frequency of the female voice was less than .6 octave above that of the male, which might have permitted discrimination on the basis of absolute properties of the acoustic stimuli. Moreover, inasmuch as transfer tests based on only segments of the vowel sounds were not conducted, the possibility of control by local features was not ruled out.

Communication among monkeys in the wild also indicates that these animals are capable of processing auditory stimuli-their natural calls-at a more complex level than might be suggested by the present results. In particular, it has been reported that monkeys are sensitive to the order in which elements of a call are arranged, analogous to syntax appreciation in human language (Robinson, 1979, in press; Snowdon, 1983). Possibly, monkeys can detect and integrate temporal order information when the acoustic elements are in their natural "vocabulary." As pointed out by Snowdon (1983), there seems to be a strong innate component to primate vocal communication, which may place limits on the range of auditory stimuli that they can process at complex levels. But even with natural calls, additional research is necessary to specify the controlling features of the acoustic stimuli, particularly in view of the evidence that intraspecific recognition of calls is, at least in birds, often based on local features of the acoustic signal (Brooks \& Falls, 1975) and that neurons in the auditory cortex of squirrel monkeys tend to respond in a similar way to species-specific calls played forward or backward (Glass \& Wollberg, 1983).

The ability to extract, store, and employ the frequency contour of complex auditory stimuli very likely is intimately related to the capacity to develop language based on acoustic signals. The necessity to recognize utterances under a wide range of transformations would seem to require such an endowment. Papousek and Papousek (1981) have discussed in detail the role of musical elements in the early ontogeny of language, pointing out the parallel development of both in the infant. At the age of 10 months, the infant in their study could imitate "proto-words and speech-like intonation contours" as well as "tones, tonal sequences, or short melodies"' (p. 194; cf. Trehub, Bull, \& Thorpe, in press). This early emergence of frequency contour discrimination in human infants contrasts sharply with the failure of our monkeys to learn the tonal structure of tunes that they had heard many thousands of times over a period of almost a year.

The issue is not that the monkey is incapable of such acoustic processing, for there is evidence based on discriminating monkey calls that suggests other- 
wise (e.g., Beecher, Petersen, Zoloth, Moody, \& Stebbins, 1979). Rather, the significant issue for comparative cognition is that the monkey's ability seems to fall so far short of that evident in young children and even in infants. Given the present results and those from related studies based on monkey vocalizations, a hypothesis worth exploring is that, unlike the case for humans, frequency contour discrimination in the monkey is largely restricted to speciesspecific acoustic signals (see Marler, 1983).

\section{REFERENCES}

Beecher, M. D., Petersen, M. R., Zoloth, S. R., Moody, D. B., \& Stebins, W. C. (1979). Perception of conspecific vocalizations by Japanese macaques: Evidence for selective attention and neural lateralization. Brain, Behavior and Evolution, 16, 443-460.

Blackwell, H. R., \& Schlosegra, H. (1943). Octave generalization, pitch discrimination, and loudness thresholds in the white rat. Journal of Experimental Psychology, 33, 407-419.

Brooks, R. J., \& FALLs, J. B. (1975). Individual recognition by song in white-throated sparrows. III. Song features used in individual recognition. Canadian Journal of Zoology, 53, 17491761 .

CowEY, A. (1968). Discrimination. In L. Weiskrantz (Ed.), Analysis of behavioral change. New York: Harper \& Row.

D'AMATo, M. R. (1973). Delayed matching and short-term memory in monkeys. In G. H. Bower (Ed.), The psychology of learning and motivation: Advances in theory and research (Vol. 7). New York: Academic Press.

D'Amato, M. R., \& Salmon, D. P. (1982). Tune discrimination in monkeys (Cebus apella) and in rats. Animal Learning \& Behavior, 10, 126-134.

Dewson, J. H., III. (1979). Toward an animal model of auditory cognitive function. In C. L. Ludlow \& M. E. Doran-Quine (Eds.), The neurological bases of language disorders in children: Methods and directions for research (NINCDS Monograph No. 22). Washington, DC: Government Printing Office.

Dewson, J. H., Pribram, K. H., \& LYNCh, J. C. (1969). Effects of temporal cortex ablation upon speech sound discrimination in the monkey. Experimental Neurology, 24, 579-591.

Glass, I., \& Wollez ra, Z. (1983). Auditory cortex responses to sequences of normal and reversed squirrel monkey vocalizations. Brain, Behavior and Evolution, 22, 13-21.

Herman, L. M. (1980). Cognitive characteristics of dolphins. In L. M. Herman (Ed.), Cetacean behavior: Mechanisms and functions. New York: Wiley.

Herman, L. M., Richards, D. G., \& Wolz, J. P. (in press). Comprehension of sentences by bottlenosed dolphins. Cognition.

Hulse, S. H., CynX, J., \& Humpal, J. (1984). Cognitive processing of pitch and rhythm structures by birds. In H. L. Roitblat, T. G. Bever, \& H. S. Terrace (Eds.), Animal cognition. Hillsdale, NJ: Erlbaum.

Marler, P. R. (1977). The structure of animal communication sounds. In T. H. Bullock (Ed.), Recognition of complex acoustic signals. Berlin: Abakon.

MArLer, P. (1983). Monkey calls: How are they perceived and what do they mean? In J. F. Eisenberg \& D. G. Kleiman (Eds.), Advances in the study of mammalian behavior. (Special publication No. 7). Shippensburg, PA: American Society of Mammalogists.

Mille R, J. D. (1977). Perception of speech sounds by animals: Evidence for speech processing by mammalian auditory mechanisms. In T. H. Bullock (Ed.), Recognition of complex acoustic signals. Berlin: Abakon.

Papouse K, M., \& Papousek, H. (1981). Musical elements in the infant's vocalization: Their significance for communication, cognition, and creativity. In L. P. Lipsitt (Ed.), Advances in infancy research (Vol. 1). Norwook, NJ: Ablex.

Robinson, J. G. (1979). An analysis of the organization of vocal communication in the titi monkey (Callicebus moloch). Zeitschrift für Tierpsychologie, 49, 381-405.

Robinson, J. G. (in press). Syntactic structures in the vocalizations of wedge capped capuchin monkeys (Cebus nigrivittatus). Behaviour.

SAles, G., \& PYe, D. (1974). Ultrasonic communication by animals. London: Chapman \& Hall.

Segal, M., \& Harrison, J. M. (1978). The control of responding by auditory stimuli: Interactions between different dimensions of the stimuli. Journal of the Experimental Analysis of Behavior, 30, 97-106.

SHEPHERD, W. T. (1911). The discrimination of articulate sounds by raccoons. American Journal of Psychology, 22, 116-119.

Shepherd, W. T. (1912). The discrimination of articulate sounds by cats. American Journal of Psychology, 23, 461-463.

Snowdon, C. T. (1983). Ethology, comparative psychology, and animal behavior. Annual Review of Psychology, 34, 63-94.

Thompson, R. K. R. (1980, July). Auditory cued reversal and matching-to-sample learning by rhesus monkeys. Paper presented at the Eighth International Congress of Primatology, Florence, Italy.

Thompson, R. K. R. (1981, October). Nonconceptual auditory matching by a rhesus monkey reflects biological constraints on cognitive processes? Paper presented at the Northeastern Meeting of the Animal Behavior Society, Kingston, Ontario.

Trehub, S. E., Buld, D. \& Thorpe, L. A. (in press). Infants' perception of melodies: The role of melodic contour. Child Development.

Wegener, J. G. (1964). Auditory discrimination behavior of normal monkeys. Journal of Auditory Research, 4, 81-106.

\section{NOTE}

1. The PET computer generates a square-wave output, and sonograms of the tunes (graciously provided by Dr. David Thomas) showed that the tunes were rich with overtones. Ultrasound, which was detected in the tunes employed by D'Amato and Salmon (1982), did not reach measureable levels in the present research.

(Manuscript received September 26, 1983; revision accepted for publication January 8, 1984.) 